This item was submitted to Loughborough's Research Repository by the author.

Items in Figshare are protected by copyright, with all rights reserved, unless otherwise indicated.

\title{
Dynapenic obesity and the risk of incident type 2 diabetes: the English Longitudinal Study of Ageing
}

PLEASE CITE THE PUBLISHED VERSION

http://dx.doi.org/10.1111/dme.12991

\section{PUBLISHER}

Wiley / @ Diabetes UK

VERSION

AM (Accepted Manuscript)

\section{PUBLISHER STATEMENT}

This work is made available according to the conditions of the Creative Commons Attribution-NonCommercialNoDerivatives 4.0 International (CC BY-NC-ND 4.0) licence. Full details of this licence are available at: https://creativecommons.org/licenses/by-nc-nd/4.0/

\section{LICENCE}

CC BY-NC-ND 4.0

\section{REPOSITORY RECORD}

Cuthbertson, Daniel J., Joshua A. Bell, S.Y. Ng, Graham J. Kemp, Mika Kivimaki, and Mark Hamer. 2019. "Dynapenic Obesity and the Risk of Incident Type 2 Diabetes: The English Longitudinal Study of Ageing". figshare. https://hdl.handle.net/2134/19363. 
4 Daniel J Cuthbertson ${ }^{1}$, BSc PhD FRCP; Joshua A Bell ${ }^{2}$, MSc; Sarah Y Ng ${ }^{1}$, MBChB MRCP;

\section{Dynapenic obesity and the risk of incident type 2 diabetes: The English Longitudinal Study of Ageing}

Graham J Kemp ${ }^{1,3}$ MA DM FRCPath; Mika Kivimaki ${ }^{2}$, PhD; Mark Hamer ${ }^{2,4}$, PhD.

${ }^{1}$ MRC-Arthritis Research UK Centre for Integrated research into Musculoskeletal Ageing, Institute of Ageing and Chronic Disease, University of Liverpool, Liverpool L9 7AL.

${ }^{2}$ Department of Epidemiology \& Public Health, University College London, 1-19 Torrington Place, London, UK, WC1E 6BT

${ }^{3}$ Musculoskeletal Biology, Institute of Ageing and Chronic Disease, University of Liverpool, Liverpool L9 7AL.

${ }^{4}$ The National Centre for Sport and Exercise Medicine, Loughborough University

Corresponding author: Daniel Cuthbertson

E-mail: daniel.cuthbertson@liverpool.ac.uk

Tel: +00 44 (0)151529 5911

Fax: +00 44 (0)151529 5888 


\section{Abstract}

Introduction Obesity is a well-established risk factor for developing type 2 diabetes mellitus (T2DM). Evidence suggests that sarcopenia, the age-related decline in muscle mass and strength, may exacerbate diabetes risk in obese individuals. The aim of this study was to determine the combined effect of obesity and low muscle strength, dynapenia, on the risk of incident T2DM in older adults.

Methods Participants were 5953 (1670 obese) men and women from the English Longitudinal Study of Ageing without known T2DM at baseline and for whom handgrip strength, biochemical and other clinical data were collected. A diagnosis of T2DM was recorded from self-reported physician diagnosis over 6 years.

Results For each unit increase in grip strength there was a reduction in diabetes risk (age and sex, BMI adjusted HR; 0.98; 95\% CI 0.96-0.99). The risk of T2DM was elevated in all obese participants, but greatest in those with low handgrip strength (HR=4.93, 95\% CI, 2.85, 8.53) compared to non-obese individuals with high handgrip strength. $11 \%$ of the sample met the threshold for weakness (handgrip strength; men $<26 \mathrm{~kg}$; women $<16 \mathrm{~kg}$ ) that was associated with elevated T2DM risk in obese $(\mathrm{HR}=3.57,95 \% \mathrm{CI}, 2.04,6.24)$ but not in non-obese $(\mathrm{HR}=$ 0.86, 95\% CI, 0.44, 1.68) compared with normal/non-obese participants.

Conclusion Dynapenic obesity, determined by high body mass index and low handgrip strength, is associated with increased risk of incident T2DM in older people. 
Sarcopenia describes a syndrome characterised by a progressive, age-related loss of skeletal muscle mass causally related to low muscle strength and impaired physical performance with recent evidence based, clinically relevant diagnostic criteria proposedski [1]. Sarcopenia confers an increased risk of physical disability [2], and death [3]. Reflecting the observations that with ageing the decline in muscle strength exceeds that of muscle mass, a distinct term dynapenia has been proposed to describe the age-related loss of muscle strength[4]. glucose disposal[5], the association between low muscle mass and dysglycemia, independently of obesity, is perhaps unsurprising $[6,7]$; there is also a clear association of sarcopenia with frank insulin resistance [8]. Furthermore, several cross-sectional studies have shown an association between handgrip strength, and features of the metabolic syndrome [6, 8]. These data implicate sarcopenia and dynapenia in the pathophysiology of insulin resistance, the metabolic syndrome and type 2 diabetes (T2DM) with advancing age.

Sarcopenic obesity, a co-occurrence of sarcopenia and obesity, is an emerging clinical entity in which these two conditions act in negative synergism in the pathophysiology of both metabolic and functional impairments. The prevalence of sarcopenic obesity depends on the definitions used, and differs considerably when sarcopenia is defined using handgrip strength $[9,10]$ or muscle mass [11-13]; Batsis et al. found that the prevalence of sarcopenic obesity definition applied [14].

In sarcopenic obesity the metabolic syndrome is more prevalent than in either obesity or sarcopenia alone [15]. There is also cross-sectional evidence to suggest that sarcopenia may exacerbate obesity-associated insulin resistance, a risk factor for T2DM [13] and that 
sarcopenic obesity is associated with poorer functional ability than either sarcopenia or obesity alone $[16,17]$. However, to our knowledge no prospective evidence is available to determine whether sarcopenia and/or dynapenia has a synergistic effect with obesity on T2DM risk, that is, whether sarcopenic/dynapenic obesity is related to a greater risk of T2DM than non-sarcopenic/dynapenic obesity.

Accordingly, the aim of this study was to utilise longitudinal population data to determine the association between low muscle strength, dynapenia, and risk of incident T2DM in lean and obese non-diabetic older individuals.

\section{Research Design and Methods}

\section{Study sample and procedures}

The English Longitudinal Study of Ageing (ELSA) is an ongoing cohort study of a nationally representative sample of the English population born on or before 29 February 1952 living in private households. The ELSA data and documentation are publicly available and can be downloaded from the UK Data Service [18]. The sample was drawn using multi-stage stratified probability sampling with postcode sectors selected at the first stage and household addresses selected at the second stage. Participants gave full, informed written consent to participate in the study and ethical approval was obtained from the London multi-centre Research Ethics Committee. Data from participants who had a body mass index (BMI) $<18.5$ $\mathrm{kg} / \mathrm{m}^{2}$ were not included in the analysis as underweight is a risk factor for mortality and these participants may have contaminated the 'normal weight' category. There were too few underweight participants $(n=48)$ to be treated as a separate group. 
94 The flow of participants is shown in Figure 1. For the purposes of the present analyses, data collected in 2004/05 (wave 2) were used as the baseline, as this was the first occasion on which clinical information was gathered $(n=10,274)$. From this cohort, 441 were excluded at baseline with a known diagnosis of diabetes. Follow-up was then at two-yearly intervals: 2006/7, 2008/9 and 2010/11. The final analytic sample consisted of 5953 individuals (1670 obese and 4283 non-obese), reflecting 57.9\% of the original baseline sample.

\section{Grip strength and anthropometric data collection}

Handgrip strength $(\mathrm{kg})$ of the dominant hand was assessed using a hand-held dynamometer, using the average of three measurements[19]. Nurses collected anthropometric data (weight, height, waist circumference). Participants’ body weight was measured using Tanita electronic scales without shoes and in light clothing, and height was measured using a Stadiometer with the Frankfurt plane in the horizontal position; body mass index (BMI) was calculated using the standard formula [weight $(\mathrm{kg}) /$ height $(\mathrm{m})$ squared]. Waist circumference $(\mathrm{cm})$ was recorded twice mid-way between the iliac crest and lower rib using measuring tape: an average of the first two measurements was used provided these differed by no more than $3 \mathrm{~cm}$; otherwise a third reading was taken and the two closest results averaged.

\section{Measurement of clinical characteristics}

Demographic and health-related questions included age, sex, cigarette smoking (current, previous, or non-smoker), the frequency of participation in vigorous, moderate, and light physical activities (more than once per week, once per week, one to three times per month, hardly ever), frequency of alcohol intake (daily, 5-6/week, 3-4/week, 1-2/week, 1-2/month, once every couple of months, 1-2/year or never). Depressive symptoms were assessed using the 8-item Centre of Epidemiological Studies Depression (CES-D) scale. Prevalent cardiovascular disease (including angina, heart disease, heart failure, heart murmur, 
arrhythmia, stroke) was assessed via self-reported physician diagnosis. Systolic and diastolic blood pressure was measured with an Omron HEM-907 blood pressure monitor three times in the sitting position after 5 min rest between each reading: the initial reading was discarded and the second and third measurements averaged.

Blood samples were obtained from a sub-sample of participants who consented and were eligible and able to give blood; this excluded men and women with clotting and bleeding disorders, or taking anti-coagulant medication. Blood samples were taken and analyzed for Creactive protein (CRP), fibrinogen, total and high-density lipoprotein (HDL) cholesterol, triglycerides, and glycated haemoglobin (HbA1c). Blood analysis was carried out at the Royal Victoria Infirmary (Newcastle-upon-Tyne, UK). Detailed information on the technicalities of the blood analysis, the internal quality control, and the external quality assessment for the laboratory have been described elsewhere [20].

\section{Incident type 2 diabetes}

Diabetes was recorded from self-reported physician diagnosis, which has been previously validated in ELSA through objective HbA1c measures[21]. Participants with known diabetes at baseline were excluded. Incident cases of diabetes were recorded at follow-up in 2006/7, 2008/9, and 2010/11; mean follow-up period 5.9 years. Participants that died, moved away from the UK, or institutionalised over follow up were censored at the date of study exit and retained in the analysis.

\section{Statistical analyses}

Characteristics of the study population at baseline were described as means (continuous variables) with standard deviations or the median with interquartile range (skewed variables), and percentages (categorical variables). ANOVA and chi-squared tests were used to test 
differences between grip strength groups. C-reactive protein was log-transformed prior to performing these tests. We used Cox proportional hazards models to compute hazard ratios (HR) with 95\% confidence intervals (CI) for the association of handgrip strength with incident diabetes. The proportional hazards assumption was examined by comparing the cumulative hazard plots grouped on the various exposure variables, and no appreciable violations were noted. The models were initially run using grip strength as a continuous

147 variable and also fitting a grip strength $\times$ BMI interaction term. A significant $(\mathrm{p}<0.001)$ interaction term between hand grip and BMI prompted us to perform these analyses stratified by BMI category (Obesity was defined as BMI $\geq 30 \mathrm{~kg} / \mathrm{m}^{2}$ and 'non-obese' defined as BMI < $30 \mathrm{~kg} / \mathrm{m}^{2}$ ). We also conducted supplementary analysis according to presence of central obesity defined as waist circumference $>102 \mathrm{~cm}$ in men and $>88 \mathrm{~cm}$ in women. Due to the strong associations between sex and grip strength we further categorized the data into sexspecific tertiles of handgrip strength, although no mediating effects of sex were observed after entering an interaction term in relation to diabetes outcome $(p=0.21)$. Age was the time scale, and for participants with no record of an event, the data were censored at wave 5 (maximum 6 years follow-up). In multivariate models we adjusted for several covariates in a step-wise fashion: Model 1 contained age and sex; Model 2 contained additional behavioural and health covariates, including smoking, alcohol, physical activity, depressive symptoms and prevalent CVD. All analyses were conducted using SPSS version 21 (IBM SPSS, 160 Armonk, NY, USA).

\section{Results}

5953 individuals (1670 obese and 4283 non-obese; 98\% white British), who were free of T2DM at baseline, completed follow-up. Of these 124 obese and 92 non-obese individuals were diagnosed with T2DM over the follow-up period. 
There were few differences in those excluded compared to those included. For example, the samples were comparable in sex distribution (\% men; $42.6 \%$ vs. $45 \%$ ) and age (65 vs. 63y). The samples differed in physical activity behaviour and mental health, with those excluded reporting higher inactivity (33\% vs. 19\%), and depressive symptoms (21\% vs. 13\%).

In men, the range of handgrip strength was 4-35.3, 35.4-44.2, $>44.2 \mathrm{~kg}$ for bottom, intermediate and top tertiles. The corresponding ranges in women were 4-19.6, 19.7-24.9, $>24.9 \mathrm{~kg}$, respectively. Table 1 shows that obese individuals in the lowest sex-specific tertile of handgrip strength were significantly older than those with either intermediate or upper tertile of handgrip strength $(\mathrm{p}<0.001)$, and were less likely to engage in any moderate or vigorous physical activity (34.4\% inactive; $\mathrm{p}<0.001)$. Despite similar smoking patterns across the three groups, the lowest handgrip strength group had a significantly higher prevalence of cardiovascular disease $(\mathrm{p}<0.001)$.

Obese participants were well matched in terms of BMI $\left(\sim 33-34 \mathrm{~kg} / \mathrm{m}^{2}\right)$ and waist circumference, across tertiles of handgrip strength (table 2). At baseline, the low handgrip strength group had significantly higher percentages of glycated haemoglobin compared to those with intermediate or high handgrip strength. Furthermore, the low handgrip strength group had higher circulating concentrations of CRP and fibrinogen compared to those with intermediate strength or low strength ( $\mathrm{p}<0.001$ and 0.009 respectively).

We assessed the linearity of the association between handgrip strength and incident diabetes in all participants by entering a squared term into the models although no deviation was noted. We observed that for each unit increase in grip strength there is a reduction in diabetes risk (age and sex, BMI adjusted HR; 0.98; 95\% CI 0.96-0.99).

The results from the analysis of T2DM risk in non-obese and obese individuals according to handgrip strength are shown in Table 3. T2DM risk did not significantly differ between the 

three tertiles of handgrip strength for non-obese participants after multivariate adjustment. The risk of T2DM was elevated in all obese participants, particularly so in those with low handgrip strength (HR=4.93, 95\% CI, 2.85, 8.53) compared to non-obese individuals with high handgrip strength after multivariate adjustment.

\section{Sensitivity analyses}

We categorised participants using sex-specific handgrip cut offs (men <26 kg; women $<16 \mathrm{~kg}$ ) for those at risk for weakness [1]. $11 \%$ of the sample met these thresholds for weakness although the pattern of results largely replicated the original results showing the same finding that sarcopenic obese were at the highest risk of incident diabetes (Table 4). In addition, we used these handgrip cut offs in combination with waist circumference as a measure of central obesity (defined as waist circumference $>102 \mathrm{~cm}$ in men and $>88 \mathrm{~cm}$ in women). When using central obesity rather than body mass index the influence of sarcopenia was less pronounced (Table 5; $\mathrm{n}=6134$ ).

\section{Discussion}

We found that obese older adults with the lowest tertile of muscle (handgrip) strength had a greater risk of developing T2DM over six years of follow-up compared with individuals of similar age and BMI with greater handgrip strength. Although adjustment for age, sex, physical activity, smoking, alcohol, depressive symptoms and prevalent cardiovascular disease partly attenuated this risk, the association persisted. In contrast, low handgrip strength did not confer a higher risk of incident T2DM in non-obese individuals. This suggests that individuals with dynapenic obesity represent a sub-group of obesity with an amplified risk of T2DM beyond that for individuals with non-dynapenic obesity.

We obtained evidence of a dose-response association, as there was a clear incremental 
increase in both incident risk of T2DM and in plasma HbA1c concentration progressively

214 from the upper to lower handgrip strength tertile. There was also a linear reduction in incident risk was not performed due to missing biochemistry data.

217 However, we did not observe significant differences in waist circumference, blood pressure, 218 lipid profile or fasting blood glucose, although low handgrip strength seemed to be accompanied by chronic low-grade inflammation, with significantly higher serum concentrations of highly sensitive CRP (hsCRP) and fibrinogen and a higher prevalence of cardiovascular disease. This association has been noted in several other epidemiological studies [22, 23] and highlights a common pathophysiological defect.

The clinical relevance of measurements of both muscle mass and muscle strength is reflected in the revised definition of sarcopenia from the Foundation for the National Institutes of Health (FNIH) Sarcopenia Project to incorporate both anatomical and functional components [1]. These newer guidelines are more clinically meaningful as they denote the functional consequences and thus relate more closely to clinical outcomes. Goodpaster et al. examining longitudinal changes in muscle mass and strength in older adults in the Health, Ageing and Body Composition Study demonstrated (annualised) rates of leg strength decline ( 3\%/year) approximately three times greater than the rates of leg lean mass ( 1\%/year) [24]. These findings clearly implicate factors other than muscle mass in determining muscle strength and these updated guidelines reflect, and take account of, this important anatomical and functional discrepancy. Thus, low grip strength is likely similarly explained by factors other than low muscle mass. Indeed, many individuals with weakness may not have low muscle mass. This had led to suggestions of a distinct term, dynapenia [25]. For practical reasons, longitudinal measurements collected in this cohort did not extend to quantification of skeletal 
muscle mass and we therefore must rely on measurements of muscle strength alone for the current study.

We chose to analyse hand grip as a continuous variable using sex-specific tertiles. In subsequent analysis we used the suggested cut points for weakness according to the FNIH criteria as previously defined (ref). However, only $11.4 \%$ of the sample met the threshold for weakness based on their handgrip (this reflects a higher prevalence of sarcopenia as a disabling condition than that observed in the FNIH project of $0.4-4 \%$ of elderly women and men) (ref) thus limiting our statistical power. Indeed, one of the limitations of using a cut point, such as the FNIH, is loss of power and information. Thus our approach of using tertiles enabled us to create equal groups across our specific sample, which helped to retain information and better examine linear trends. Interestingly, whichever thresholds for weakness were applied the association between weakness and incident T2DM remained the same for the obese participants.

In many T2DM patients with advancing age, severe obesity and comorbidities including cardiovascular disease and osteoarthritis aerobic exercise is not feasible. In contrast, resistance training provides an effective exercise alternative by increasing muscle mass and strength (and thus counteracting age- and disease-related skeletal muscle loss and muscle weakness), in visceral fat deposition and improvements in insulin sensitivity and glycemic control [26, 27]. Thus our findings are biologically plausible. Skeletal muscle represents a major organ for glucose homeostasis, responsible for up to $75 \%$ of post-prandial (i.e. insulinstimulated) glucose uptake [5]. Low muscle mass might therefore be expected to impair glucose homeostasis. There is epidemiological evidence in obese, older individuals to link sarcopenia with the metabolic syndrome and with T2DM [28], although it is not possible to infer temporality between these conditions from cross-sectional data. Longitudinal studies have shown an accelerated decline in muscle mass over several years in patients already 
263 Although those with low handgrip strength were less likely to be physically active, statistical adjustment for physical activity did not alter the predominant relationship, suggesting that the increased risk of T2DM in weaker individuals does not merely reflect more sedentary behaviour. Evidence from human and transgenic animal models that interventions influencing skeletal muscle growth and/or metabolism are protective against metabolic diseases may provide some further mechanistic insight: preservation of oxidative capacities in aged muscles prevents muscle loss, reduces intramyocellular lipid accumulation and protects against metabolic diseases [30]; up-regulation of PGC1- $\alpha$, a master regulator of mitochondrial metabolism, protects against age-related muscle loss and improves insulin resistance [31]; and importantly, in patients with T2DM resistance exercise, a potent anabolic stimulus for skeletal muscle, significantly improves glycaemic control [32].

We acknowledge some limitations of this study. The nature of large-scale, population-based longitudinal studies makes sample attrition and incomplete data collection inevitable. Biochemical measurements were only available for 4186 from a total of 5953 participants, and this may be a source of selection bias, with participants able to provide samples likely to be in better health. Although we saw that for each unit increase in grip strength there is a reduction in diabetes risk (HR 0.98; 95\% CI 0.96-0.99), the missing biochemical data made analysis of the relationship of grip strength with $\mathrm{HbA}_{1} \mathrm{c}$ impractical.

A further limitation is the use of questionnaires to ascertain diagnosis of T2DM, although self-reported physician-diagnosed diabetes has been well validated with HbA1c in the ELSA sample. Since self-reported physician diagnosis of diabetes cannot account for undetected cases, our results might be biased towards the obese sample who may be more likely to be diagnosed because of more frequent contact with health services to treat other risk factors. 
Furthermore this potentially could lead to reverse causation with undiagnosed T2DM at

287

288 baseline being associated with low grip strength. Formal assessment using physical activity monitors and biannual glucose tolerance tests might have afforded further mechanistic insight, particularly with respect to the domains of physical activity and degrees of insulin resistance. Our sample was also predominantly white-European, and results may therefore not directly apply to older adults of other ethnic groups, such as those of black ethnicities.

In conclusion, the risk of developing T2DM was significantly increased in older obese adults with low muscle strength, a finding that cannot be fully explained by levels of physical activity or other risk factors. Our findings suggest that dynapenic obesity in older adults represents a form of obesity which is related to particularly high risk of T2DM. Further research is needed to examine whether strategies to increase muscle strength would reduce diabetes risk in obese older adults.

\section{Author Contributions}

All authors were responsible for the conception, design and drafting of the manuscript and approved the final version for publication.

\section{Acknowledgements}

A team of researchers based at University College London, the Institute of Fiscal Studies and the National Centre for Social Research developed the English Longitudinal Study of Ageing (ELSA), whose data were made available through the UK Data Archive. Funding for ELSA is provided by the National Institute on Aging in the United States (grants 2RO1AG764401A1 and 2RO1AG017644) and a consortium of UK government departments coordinated by the Office for National Statistics. JAB is supported by an Economic and Social Research 
310 Council (ESRC) studentship, MK by the Medical Research Council (K013351) and the

311 Economic and Social Research Council, MH by the British Heart Foundation

312 (RE/10/005/28296), and GJK and DJC by the Medical Research Council and Arthritis

313 Research UK (MR/ K006312/1) as part of the MRC - Arthritis Research UK Centre for

314 Integrated Research into Musculoskeletal Ageing (CIMA). The funders had no role in the

315 study design, in the collection, analysis and interpretation of data, in writing of the report or

316 in the decision to submit the paper for publication. The developers and funders of ELSA and

317 the Archive do not bear any responsibility for the analyses or interpretations presented here.

319 Conflicts of interest

320 None of the authors have any competing interests to declare. 


\section{References}

1. Studenski SA, Peters KW, Alley DE, Cawthon PM, McLean RR, Harris TB, et al. The FNIH sarcopenia project: rationale, study description, conference recommendations, and final estimates. J Gerontol A Biol Sci Med Sci. United States 2014: 547-558.

2. Janssen I, Heymsfield SB, Ross R. Low relative skeletal muscle mass (sarcopenia) in older persons is associated with functional impairment and physical disability. J Am Geriatr Soc. United States 2002: 889-896.

3. Vetrano DL, Landi F, Volpato S, Corsonello A, Meloni E, Bernabei R, et al. Association of sarcopenia with short- and long-term mortality in older adults admitted to acute care wards: results from the CRIME study. J Gerontol A Biol Sci Med Sci 2014; 69:1154-1161.

4. Clark BC, Manini TM. Sarcopenia =/= dynapenia. J Gerontol A Biol Sci Med Sci 2008; 63:829-834.

5. DeFronzo RA, Tripathy D. Skeletal Muscle Insulin Resistance Is the Primary Defect in Type 2 Diabetes. Diabetes Care 2009; 32:S157-S163.

6. Sayer AA, Syddall HE, Dennison EM, Martin HJ, Phillips DI, Cooper C, et al. Grip strength and the metabolic syndrome: findings from the Hertfordshire Cohort Study. QJM : monthly journal of the Association of Physicians 2007; 100:707-713.

7. Srikanthan P, Hevener AL, Karlamangla AS. Sarcopenia exacerbates obesityassociated insulin resistance and dysglycemia: findings from the National Health and Nutrition Examination Survey III. PloS one 2010: e10805.

8. Srikanthan P, Karlamangla AS. Relative muscle mass is inversely associated with insulin resistance and prediabetes. Findings from the third National Health and Nutrition Examination Survey. J Clin Endocrinol Metab 2011; 96:2898-2903. 
9. Schrager MA, Metter EJ, Simonsick E, Ble A, Bandinelli S, Lauretani F, et al.

Sarcopenic obesity and inflammation in the InCHIANTI study. J Appl Physiol (1985) 2007; 102:919-925.

10. Visser M, van Venrooij LM, Vulperhorst L, de Vos R, Wisselink W, van Leeuwen

PA, et al. Sarcopenic obesity is associated with adverse clinical outcome after cardiac surgery. Nutr Metab Cardiovasc Dis 2013; 23:511-518.

11. Baumgartner RN. Body composition in healthy aging. Ann N Y Acad Sci 2000; 904:437-448.

12. Chung JY, Kang HT, Lee DC, Lee HR, Lee YJ. Body composition and its association with cardiometabolic risk factors in the elderly: a focus on sarcopenic obesity. Arch Gerontol Geriatr 2013; 56:270-278.

13. Srikanthan P, Hevener AL, Karlamangla AS. Sarcopenia exacerbates obesityassociated insulin resistance and dysglycemia: findings from the National Health and Nutrition Examination Survey III. PloS one 2010; 5:e10805.

14. Batsis JA, Barre LK, Mackenzie TA, Pratt SI, Lopez-Jimenez F, Bartels SJ. Variation in the prevalence of sarcopenia and sarcopenic obesity in older adults associated with different research definitions: dual-energy X-ray absorptiometry data from the National Health and Nutrition Examination Survey 1999-2004. J Am Geriatr Soc 2013; 61:974-980. 15. Lim S, Kim JH, Yoon JW, Kang SM, Choi SH, Park YJ, et al. Sarcopenic obesity: prevalence and association with metabolic syndrome in the Korean Longitudinal Study on Health and Aging (KLoSHA). Diabetes care 2010; 33:1652-1654. 16. Baumgartner RN, Wayne SJ, Waters DL, Janssen I, Gallagher D, Morley JE. Sarcopenic obesity predicts instrumental activities of daily living disability in the elderly. Obesity research 2004; 12:1995-2004. 
372 17. Rolland Y, Lauwers-Cances V, Cristini C, Abellan van Kan G, Janssen I, Morley JE, 373 et al. Difficulties with physical function associated with obesity, sarcopenia, and sarcopenic374 obesity in community-dwelling elderly women: the EPIDOS (EPIDemiologie de 375 l'OSteoporose) Study. The American journal of clinical nutrition 2009; 89:1895-1900.

376 18. UK_Data_Archive. ELSA user guide and documentation. 2008.

19. Hamer M, Stamatakis E. Screen-based sedentary behavior, physical activity, and muscle strength in the English longitudinal study of ageing. PLoS One 2013; 8:e66222. 20. Graig R, Deverill C, Pickering K. Quality control of blood, saliva and urine analytes. In: Spronston K, J Mindell, eds. Health Survey for England 2004, Methodology and Documentation. London: The Information Centre 2006: 34-41.

21. Smith L, Hamer M. Television viewing time and risk of incident diabetes mellitus: the English Longitudinal Study of Ageing. Diabet Med 2014; 31:1572-1576.

22. Visser M, Pahor M, Taaffe DR, Goodpaster BH, Simonsick EM, Newman AB, et al. Relationship of interleukin-6 and tumor necrosis factor-alpha with muscle mass and muscle strength in elderly men and women: the Health ABC Study. J Gerontol A Biol Sci Med Sci 2002; 57:M326-332.

23. Taaffe DR, Harris TB, Ferrucci L, Rowe J, Seeman TE. Cross-sectional and prospective relationships of interleukin-6 and C-reactive protein with physical performance in elderly persons: MacArthur studies of successful aging. J Gerontol A Biol Sci Med Sci 2000; 55:M709-715.

24. Goodpaster BH, Park SW, Harris TB, Kritchevsky SB, Nevitt M, Schwartz AV, et al. The loss of skeletal muscle strength, mass, and quality in older adults: the health, aging and body composition study. J Gerontol A Biol Sci Med Sci 2006; 61:1059-1064. 25. Clark BC, Manini TM. Functional consequences of sarcopenia and dynapenia in the elderly. Curr Opin Clin Nutr Metab Care 2010; 13:271-276. 

short-term circuit weight training program on glycaemic control in NIDDM. Diabetes Res Clin Pract 1998; 40:53-61.

400 27. Eves ND, Plotnikoff RC. Resistance training and type 2 diabetes: Considerations for 401 implementation at the population level. Diabetes Care 2006; 29:1933-1941.

402 28. Park SW, Goodpaster BH, Strotmeyer ES, de Rekeneire N, Harris TB, Schwartz AV, 403 et al. Decreased muscle strength and quality in older adults with type 2 diabetes: the health, aging, and body composition study. Diabetes 2006; 55:1813-1818.

29. Park SW, Goodpaster BH, Lee JS, Kuller LH, Boudreau R, de Rekeneire N, et al.

406 Excessive loss of skeletal muscle mass in older adults with type 2 diabetes. Diabetes care $407 \quad 2009 ; 32: 1993-1997$.

30. Harrison BC, Leinwand LA. Fighting fat with muscle: bulking up to slim down. Cell 409 metabolism 2008; 7:97-98.

31. Wenz T, Rossi SG, Rotundo RL, Spiegelman BM, Moraes CT. Increased muscle PGC-1alpha expression protects from sarcopenia and metabolic disease during aging.

412 Proceedings of the National Academy of Sciences of the United States of America 2009; 106:20405-20410. diabetes: bring on the heavy weights. Diabetes care 2003; 26:1580-1588. 
Figure 1 Flow chart describing the selection of participants for the present study.

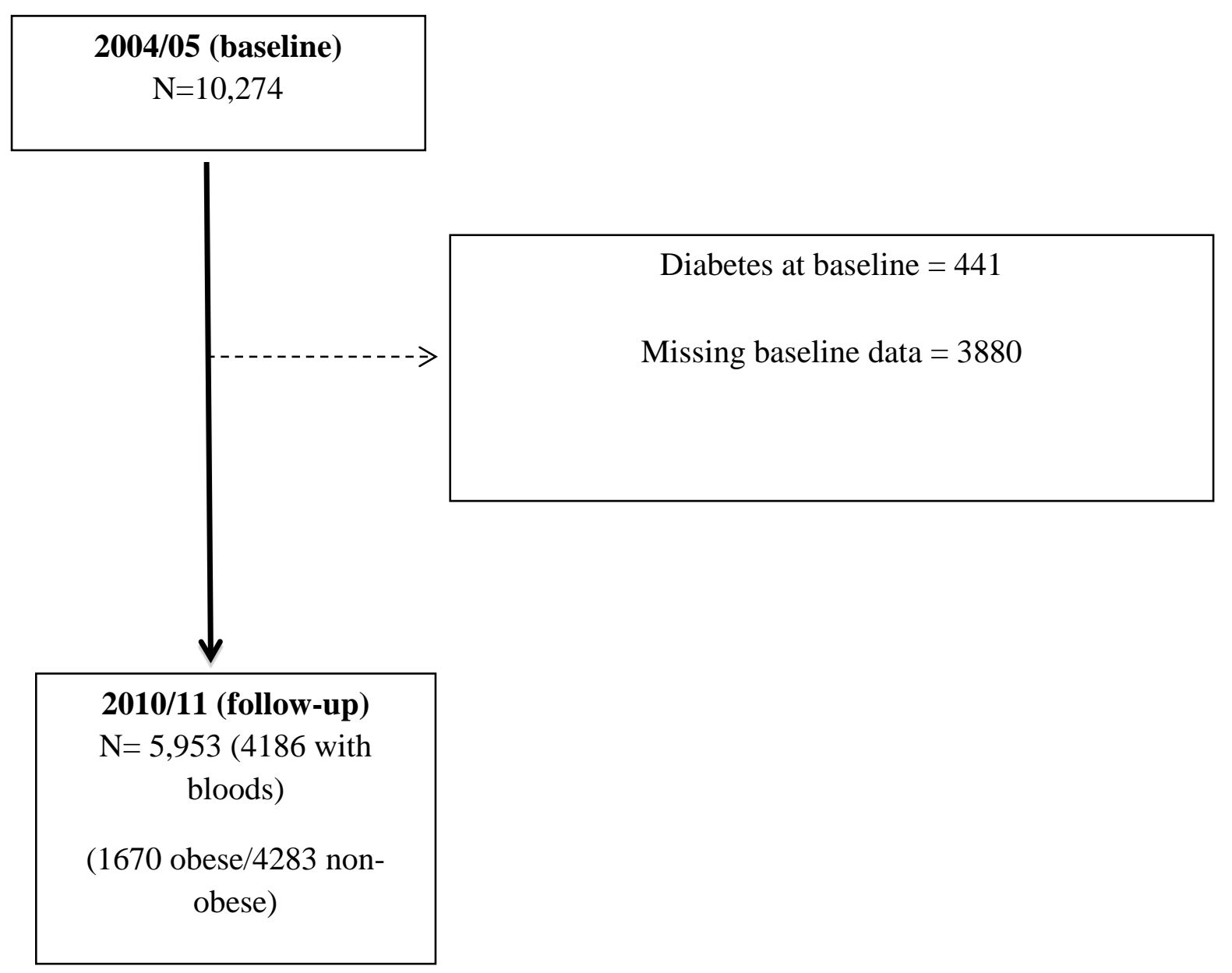


Table 1 Demographic characteristics of the sample in relation to grip strength ( $\mathrm{n}=5953)$

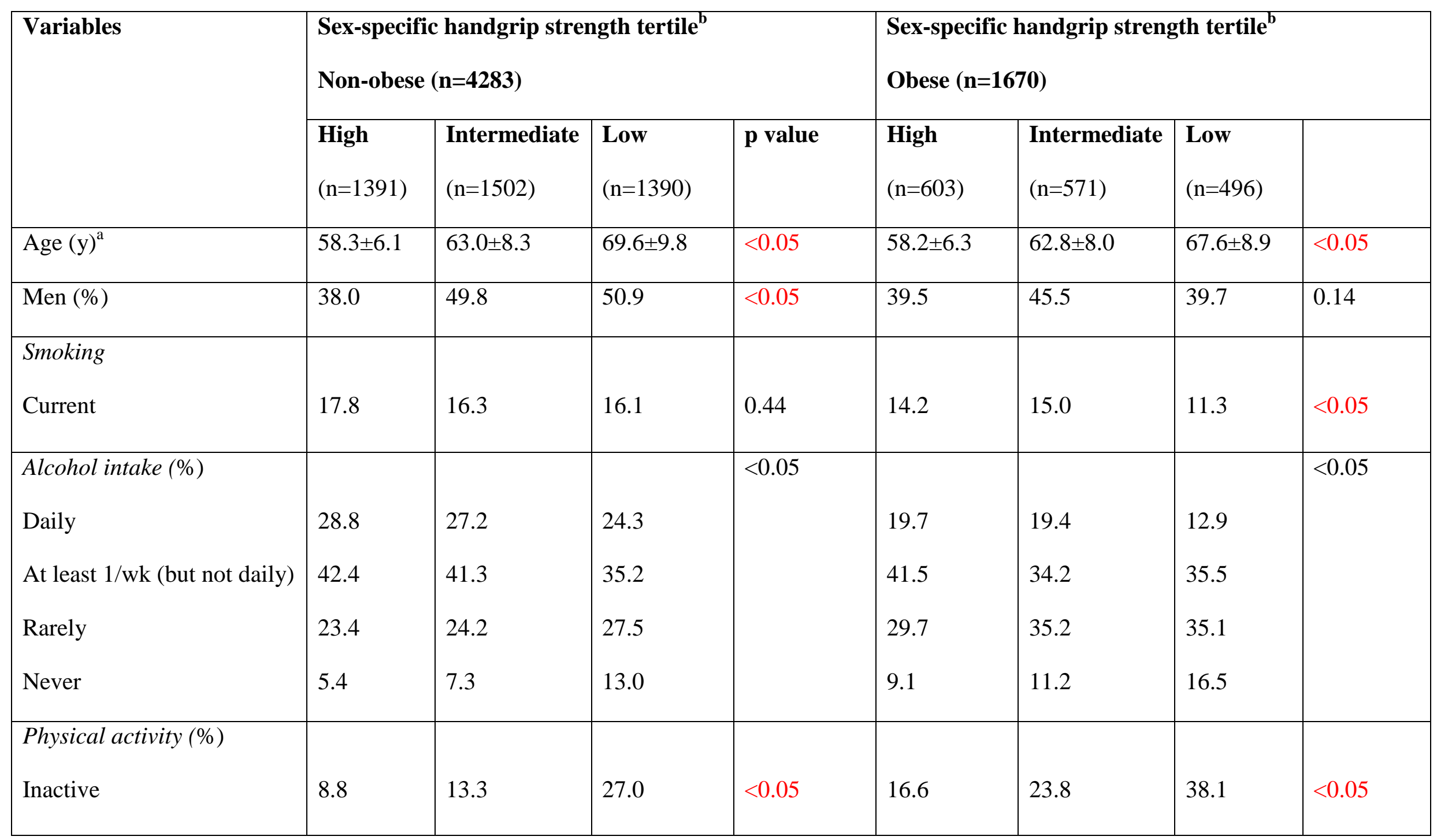




\begin{tabular}{|l|l|l|l|l|l|l|l|l|}
\hline Moderate (at least 1/week) & 48.5 & 51.4 & 52.2 & 55.6 & 51.5 & 43.8 & 27.9 & 24.7 \\
Vigorous (at least 1/week) & 42.8 & 35.3 & 20.8 & & & & \\
\hline Depressive symptoms (\%) & 9.3 & 10.9 & 16.3 & $<0.05$ & 12.4 & 13.3 & 17.9 & $<0.05$ \\
\hline Prevalent CVD (\%) & 11.3 & 12.9 & 15.8 & $<0.05$ & 10.8 & 14.5 & 15.9 \\
\hline
\end{tabular}

${ }^{\mathrm{a}}$ Data presented as mean \pm SD

${ }^{\mathrm{b}}$ In men, the range of handgrip strength was $4-35.3$, 35.4 - 44.2, $>44.2 \mathrm{~kg}$ for bottom, intermediate and top tertile. The corresponding ranges in women were 4 - 19.6, 19.7 - 24.9, $>24.9 \mathrm{~kg}$, respectively. 
Table 2 Sarcopenic obesity and biomarkers_in the 4,186 individuals in whom biochemical data were available.

\begin{tabular}{|c|c|c|c|c|c|c|c|c|}
\hline \multirow[t]{2}{*}{ Variable } & \multicolumn{4}{|c|}{$\begin{array}{l}\text { Sex-specific handgrip strength tertile }{ }^{\mathrm{a}} \\
\text { Non-obese }\end{array}$} & \multicolumn{4}{|c|}{$\begin{array}{l}\text { Sex-specific handgrip strength tertile }{ }^{a} \\
\text { Obese }\end{array}$} \\
\hline & $\begin{array}{l}\text { High } \\
(n=1082)\end{array}$ & $\begin{array}{l}\text { Intermediate } \\
(n=1085)\end{array}$ & $\begin{array}{l}\text { Low } \\
(n=908)\end{array}$ & p value & $\begin{array}{l}\text { High } \\
\text { (424) }\end{array}$ & $\begin{array}{l}\text { Intermediate } \\
(n=380)\end{array}$ & $\begin{array}{l}\text { Low } \\
(n=307)\end{array}$ & p value \\
\hline Body mass index $\left(\mathrm{kg} / \mathrm{m}^{2}\right)$ & $25.8 \pm 2.5$ & $25.7 \pm 2.6$ & $25.2 \pm 2.7$ & $<0.001$ & $33.9 \pm 3.8$ & $33.4 \pm 3.6$ & $33.6 \pm 3.3$ & 0.38 \\
\hline Waist circumference $(\mathrm{cm})$ & $89.0 \pm 11.6$ & $90.4 \pm 12.2$ & $89.6 \pm 13.6$ & 0.005 & $105.3 \pm 16.0$ & $105.6 \pm 16.0$ & $105.5 \pm 13.1$ & 0.78 \\
\hline Systolic blood pressure (mmHg) & $131.2 \pm 16.9$ & $133.5 \pm 18.9$ & $134.6 \pm 19.1$ & $<0.001$ & $138.1 \pm 17.1$ & $138.6 \pm 18.2$ & $139.5 \pm 17.7$ & 0.61 \\
\hline $\begin{array}{l}\text { Glycated haemoglobin (\%) } \\
\text { Glycated haemoglobin (mmol/mol) }\end{array}$ & $\begin{array}{l}5.4 \pm 0.5 \\
35 \pm 5\end{array}$ & $\begin{array}{l}5.5 \pm 0.5 \\
36 \pm 5\end{array}$ & $\begin{array}{l}5.5 \pm 0.6 \\
37 \pm 6\end{array}$ & $<0.001$ & $\begin{array}{l}5.6 \pm 0.6 \\
38 \pm 8\end{array}$ & $\begin{array}{l}5.6 \pm 0.5 \\
38 \pm 6\end{array}$ & $\begin{array}{l}5.8 \pm 0.8 \\
40 \pm 9\end{array}$ & 0.034 \\
\hline Total cholesterol (mmol/l) & $6.17 \pm 1.21$ & $6.02 \pm 1.31$ & $5.79 \pm 1.19$ & $<0.001$ & $6.16 \pm 1.21$ & $5.90 \pm 1.31$ & $5.81 \pm 1.19$ & $<0.001$ \\
\hline HDL cholesterol (mmol/l) & $1.62 \pm 0.33$ & $1.58 \pm 0.33$ & $1.55 \pm 0.32$ & $<0.001$ & $1.44 \pm 0.33$ & $1.42 \pm 0.33$ & $1.42 \pm 0.32$ & 0.26 \\
\hline Triglycerides (mmol/l) & $1.64 \pm 1.33$ & $1.64 \pm 1.12$ & $1.63 \pm 1.23$ & 0.80 & $2.08 \pm 1.33$ & $2.03 \pm 1.12$ & $2.02 \pm 1.23$ & 0.58 \\
\hline C-reactive protein $(\mathrm{mg} / \mathrm{l})^{\mathrm{b}}$ & $1.3(1.9)$ & \begin{tabular}{|l|}
$1.6(2.4)$ \\
\end{tabular} & $1.9(2.8)$ & $<0.001$ & 3.3 (3.7) & $3.0(4.2)$ & $3.8(5.4)$ & $<0.001$ \\
\hline Fibrinogen (g/l) & $2.94 \pm 0.67$ & $3.09 \pm 0.70$ & $3.23 \pm 0.67$ & $<0.001$ & $3.24 \pm 0.67$ & $3.28 \pm 0.70$ & $3.39 \pm 0.67$ & $<0.001$ \\
\hline
\end{tabular}

Data presented as mean \pm SD unless otherwise stated; 
${ }^{\mathrm{a}}$ In men, the range of handgrip strength was $4-35.3,35.4-44.2,>44.2 \mathrm{~kg}$ for bottom, intermediate and top tertile. The corresponding ranges in women were $4-19.6,19.7-24.9,>24.9 \mathrm{~kg}$, respectively.

bmedian (interquartile range). 
Table 3 Associations between handgrip strength and risk of incident type 2 diabetes among obese and non-obese participants free of physician diagnosed diabetes at baseline $(n=5,953)$.

\begin{tabular}{|l|l|l|l|}
\hline $\begin{array}{l}\text { Sex-specific handgrip } \\
\text { strength tertile }\end{array}$ & Cases/n & $\begin{array}{l}\text { Model 1 } \\
\text { HR (95\% CI) }\end{array}$ & $\begin{array}{l}\text { Model 2 } \\
\text { HR (95\% CI) }\end{array}$ \\
\hline Non-obese participants & & & \\
\hline High & $21 / 1391$ & 1.00 (reference) & 1.00 (reference) \\
\hline Intermediate & $36 / 1502$ & $1.54(0.90,2.63)$ & $1.49(0.86,2.53)$ \\
\hline Low & $35 / 1390$ & $1.72(0.98,3.02)$ & $1.51(0.86,2.66)$ \\
\hline Obese participants & & & \\
\hline High & $33 / 603$ & $3.47(2.03,5.96)$ & $3.25(1.89,5.60)$ \\
\hline Intermediate & $49 / 571$ & $5.68(3.42,9.45)$ & $4.85(2.90,8.11)$ \\
\hline Low & $42 / 496$ & $5.97(3.49,10.23)$ & $4.93(2.85,8.53)$ \\
\hline
\end{tabular}

Model 1: adjusted for age and sex. Model 2: adjusted for age, sex, physical activity, smoking, alcohol, depressive symptoms, prevalent cardiovascular disease (angina, heart disease, heart failure, heart murmur, arrhythmia, stroke) 
Table 4 Associations between handgrip strength using a binary cut off point with weakness defined as handgrip $<26 \mathrm{~kg}$ in men and $<16 \mathrm{~kg}$ in women and risk of incident type 2 diabetes among obese and non-obese participants free of physician diagnosed diabetes at baseline ( $\mathrm{n}=5953)$.

\begin{tabular}{|l|l|l|l|}
\hline $\begin{array}{l}\text { Sex-specific handgrip } \\
\text { strength using binary cut * }\end{array}$ & Cases/n & Model 1 & $\begin{array}{l}\text { Model 2 } \\
\text { HR (95\% CI) }\end{array}$ \\
\hline Non-obese participants & & & \\
\hline Normal & $82 / 3789$ & 1.00 (reference) & 1.00 (reference) \\
\hline Weak & $10 / 494$ & $0.97(0.49,1.90)$ & $0.86(0.44,1.68)$ \\
\hline Obese participants & & & \\
\hline Normal & $108 / 1484$ & $3.43(2.57,4.57)$ & $3.13(2.33,4.18)$ \\
\hline Weak & $16 / 186$ & $4.27(2.47,7.37)$ & $3.57(2.04,6.24)$ \\
\hline
\end{tabular}

*Weakness defined as handgrip $<26 \mathrm{~kg}$ in men and $<16 \mathrm{~kg}$ in women.

Model 1: adjusted for age and sex.

Model 2: adjusted for age, sex, physical activity, smoking, alcohol, depressive symptoms, and prevalent cardiovascular disease (angina, heart disease, heart failure, heart murmur, arrhythmia, stroke) 
Table 5 Associations between handgrip strength and risk of incident type 2 diabetes among centrally obese and non-obese participants free of physician diagnosed diabetes at baseline $(\mathrm{n}=6134)$.

\begin{tabular}{|l|l|l|l|}
\hline $\begin{array}{l}\text { Sex-specific handgrip } \\
\text { strength using binary cut } *\end{array}$ & Cases/n & $\begin{array}{l}\text { Model 1 } \\
\text { HR (95\% CI) }\end{array}$ & $\begin{array}{l}\text { Model 2 } \\
\text { HR (95\% CI) }\end{array}$ \\
\hline Non-obese participants & & & \\
\hline Normal & $37 / 2656$ & 1.00 (reference) & 1.00 (reference) \\
\hline Weak & $7 / 388$ & $1.51(0.66,3.43)$ & $1.32(0.58,3.00)$ \\
\hline Obese participants $\dagger$ & & & \\
\hline Normal & $158 / 2742$ & $4.39(3.07,6.28)$ & $4.09(2.85,5.87)$ \\
\hline Weak & $19 / 348$ & $4.82(2.72,8.55)$ & $3.99(2.23,7.13)$ \\
\hline
\end{tabular}

*Weakness defined as handgrip $<26 \mathrm{~kg}$ in men and $<16 \mathrm{~kg}$ in women.

†Central obesity defined as waist circumference $>102 \mathrm{~cm}$ in men and $>88 \mathrm{~cm}$ in women

Model 1: adjusted for age and sex.

Model 2: adjusted for age, sex, physical activity, smoking, alcohol, depressive symptoms, and prevalent cardiovascular disease (angina, heart disease, heart failure, heart murmur, arrhythmia, stroke) 